\title{
Sample Sufficiency for Estimation of the Mean of Rye Traits at Flowering Stage
}

\author{
Cirineu Tolfo Bandeira ${ }^{1}$, Alberto Cargnelutti Filho ${ }^{2}$, Fernanda Carini ${ }^{1}$, Denison Esequiel Schabarum ${ }^{1}$, \\ Jéssica Andiara Kleinpaul ${ }^{1}$ \& Rafael Vieira Pezzini ${ }^{1}$ \\ ${ }^{1}$ Postgraduate Program in Agronomy, Federal University of Santa Maria, Santa Maria, Brazil \\ ${ }^{2}$ Department of Crop Science, Federal University of Santa Maria, Santa Maria, Brazil \\ Correspondence: Alberto Cargnelutti Filho, Department of Crop Science, Federal University of Santa Maria, \\ Avenida Roraima, $\mathrm{n}^{\circ}$ 1000, Bairro Camobi, CEP 97105-900, Santa Maria, RS, Brazil. Tel: 55-55-3220-8899. \\ E-mail: alberto.cargnelutti.filho@gmail.com
}

Received: December 14, 2017

Accepted: January 15, 2018

Online Published: February 15, 2018

doi:10.5539/jas.v10n3p178

URL: https://doi.org/10.5539/jas.v10n3p178

\begin{abstract}
The objective of this study was to determine the sample size to estimate the traits mean in cultivars and sowing times, at flowering of rye culture. Ten uniformity trials were performed combining two cultivars in five sowing times. In the flowering of culture, in 100 plants of each uniformity trial, eleven traits were evaluated. The descriptive statistics was calculated and it was determined the sample size to estimate the mean in levels of precision (amplitude of the confidence interval of $95 \%$ for $5,10, \ldots, 35 \%$ of the mean) by resampling with replacement. The cob length presented the lowest variability among the eleven traits and, consequently, smaller sample size in both cultivars and five sowing time. There is variability in the sample size to estimate the mean among the traits, cultivars and sowing times. The measurement of 425, 276, 189 and 138 plants in cultivar BRS Progresso and 642, 413, 285 and 211 plants in cultivar Temprano, are enough to estimate the mean amplitude of the confidence interval of $95 \%$ maximum of 20, 25, 30 and 35\%, respectively, for all the traits and sowing times.
\end{abstract}

Keywords: experimental planning, Secale cereale L., resampling

\section{Introduction}

The rye culture is used for various purposes, among them in human food, as cover crop and forage for animals. As plant coverage, it plays an important role in the management of the agricultural sector, since it reduces nitrogen loss (Martinez-Feria, Dietzel, Liebman, Helmers, \& Archontoulis, 2016), increases the retained water available in the soil (Basche et al., 2016) and assists in the control of soil erosion (Pantoja, Woli, Sawyer, \& Barker, 2016).

Experiment is a study previously planned, which follows the basic principles of repetition, randomization and local control, in which comparisons are made of the effects of treatments. The experiment must consider the researcher's interests and the basic assumptions required for the validity of the statistical analysis (Banzatto \& Kronka, 2013). During the execution of an experiment, often, for the evaluation of the traits it is not possible to sample the experimental unit in its entirety and, in this situation, it is recommended to use a representative sample of the experimental unit (Storck, Garcia, Lopes, \& Estefanel, 2016).

The correct sample sizing is important for obtaining estimates with desired precision, optimization of labor, time and resources of the researcher. The sample size is directly proportional to the variability of the data and the degree of confidence desired, the later determined by the researcher (Bussab \& Morettin, 2013). In addition, the sample size must be planned appropriately to validate the research (Brito, Grigoletto, Nóbrega, \& Córdova, 2016), because it interferes in the estimates of distributions of statistics of interest (Ramírez, Barrera, \& Correa, 2013).

The sample size was studied in crops, such as soybeans (Cargnelutti Filho, Evangelista, Gonçalves, \& Storck, 2009), maize (Toebe, Cargnelutti Filho, Burin, Casarotto, \& Haesbaert, 2014), forage turnip (Cargnelutti Filho et al., 2014), black oats (Cargnelutti Filho et al., 2015b) and sunflower (Silva, Santos, Oliveira, Sousa, \& Fernandes, 2015). The objective of this study was to determine the sample size to estimate the traits mean in cultivars and sowing times, at flowering of rye culture. 


\section{Material and Methods}

Ten uniformity trials were carried out, in the harvest of 2016, in the experimental area of the Crop Science Department of the Federal University of Santa Maria, Rio Grande do Sul with the cultivation of rye. The climate of the region, according to the classification of Köppen, is of Cfa humid subtropical type with hot summers and without a defined dry season (Heldwein, Buriol, \& Streck, 2009) and the soil is classified as Paleudalf (Santos, Almeida, \& Oliveira, 2013).

Uniformity trials were performed with two cultivars in five sowing times. The cultivars were BRS Progresso, intended for the production of grains, and Temprano, recommended as cover crop and grazing. The five sowing times were: May 3, 2016 (time 1), May 25, 2016 (time 2), June 7, 2016 (time 3), June 22, 2016 (time 4) and July 4, 2016 (time 5). In each sowing, the soil was prepared in a conventional manner, with light harrow, basic fertilization of $25 \mathrm{~kg} \mathrm{ha}^{-1}$ of nitrogen $(\mathrm{N}), 100 \mathrm{~kg} \mathrm{ha}^{-1}$ of phosphor (P), $100 \mathrm{~kg} \mathrm{ha}^{-1}$ of potassium (K) and broadcasting with a density of 455 seeds $\mathrm{m}^{-2}$.

In the first time, each cultivar was sown in an area of $320 \mathrm{~m}^{2}(20 \mathrm{~m} \times 16 \mathrm{~m})$ and at other sowing times, each cultivar occupied $375 \mathrm{~m}^{2}(25 \mathrm{~m} \times 15 \mathrm{~m})$. The crops treatments were performed uniformly throughout the experimental area. In the central area of each uniformity trial, a grid with 100 sampling points spaced from $1 \mathrm{~m} \times$ $1 \mathrm{~m}$ was demarcated, with stakes, forming an array of ten rows and ten columns. It was chosen randomly, one plant per sampling point, and in the flowering of plants, the following traits were evaluated: number of tillers, obtained by counting the number of stalks except the main stalk (NT); number of ears (NE); length of ear (LE, in $\mathrm{cm}$ ); fresh matter of leaf (FML, in $\mathrm{g}$ ); fresh matter of stalk (FMS, in g); fresh matter of ear (FME, in g); fresh matter of plant (FMP = FML + FMS + FME, in g); dry matter of leaf (DML, in g); dry matter of stalk (DMS, in $\mathrm{g}$ ); dry matter of ear (DME, in g); and dry matter of plant (DMP = DML + DMS + DME, in g).

For each trait, cultivation and sowing time, measures of central tendency, variability, of asymmetry and kurtosis were calculated and the data normality was verified by the Kolmogorv-Smirnov test and the randomness by run test (Campos, 1983). For each trait, cultivation and sowing time, 999 sample sizes were planned, whose initial size was of two plants and the others were obtained with the plant growth. Thus, planned sample sizes were 2,3 , $4, \ldots 1,000$ plants.

For each sample size planned, 10,000 resamplings were performed with replacement. In each resampling the mean was calculated. Based on 10,000 estimates of the mean, the percentile $2.5 \%$ and the percentile $97.5 \%$ were calculated. The amplitude of the confidence interval of $95 \%\left(\mathrm{ACI}_{95 \%}\right)$ was calculated, by the difference between the percentile $97.5 \%$ and percentile $2.5 \%$.

For the determination of sample size (number of plants) required to estimate the average, maximum residue limits were fixed of $\mathrm{ACI}_{95 \%}$ at $5,10,15,20,25,30$ and $35 \%$ of the mean. Then, from the size of the initial sample ( $\mathrm{n}=$ two plants), and is considered as an appropriate sample size $(\mathrm{n})$ the number of plants from which the $\mathrm{ACI}_{95 \%}$ was less than or equal to the maximum limit established for each level of precision.

Graphically, it was represented the $2.5 \%$ percentile, the mean and $97.5 \%$ percentile, of the trait with the highest and lowest estimated sample size in both cultivars, for some of the planned sample sizes planned $(\mathrm{n}=10,20, \ldots$, 1,000 plants). Statistical analyzes were performed with the aid of the program R (R Core Team, 2017) and the application Microsoft Office Excel®.

\section{Results and Discussion}

The sowing time one and five showed the lowest averages for the traits, in both cultivars (Tables 1 and 2), demonstrating that the means were influenced by the sowing time. The mean values were higher than the median in cultivars BRS Progresso and Temprano in the majority of the traits in sowing times. This is due to the fact that some of the 100 plants evaluated presented high values of these traits, contributing to the asymmetric displacement to the right (Bussab \& Morettin, 2013). 
Table 1. Minimum (min), maximum (max), mean, median (med), standard deviation (sd), standard error (se), coefficient of variation $(\mathrm{CV}$, in \%), asymmetry $(\mathrm{A})$, kurtosis $(\mathrm{K})$ and p-value of the traits: number of tillers (NT); number of ears (NE); length of ear (LE, in cm); fresh matter of leaf (FML, in $\mathrm{g}$ ); fresh matter of stalk (FMS, in $\mathrm{g})$; fresh matter of ear (FME, in g); fresh matter of plant (FMP = FML + FMS + FME, in g); dry matter of leaf $(\mathrm{DML}$, in $\mathrm{g})$; dry matter of stalk (DMS, in $\mathrm{g})$; dry matter of ear (DME, in g); and dry matter of plant (DMP = $\mathrm{DML}+\mathrm{DMS}+\mathrm{DME}$, in $\mathrm{g})$ in the sowing times of rye BRS Progresso

\begin{tabular}{|c|c|c|c|c|c|c|c|c|c|c|c|}
\hline Statistic & NT & $\mathrm{NE}$ & LE & FML & FMS & FME & FMP & DML & DMS & DME & DMP \\
\hline \multicolumn{12}{|c|}{ Sowing time 1 (May 3, 2016) } \\
\hline $\min$ & 0.00 & 0.00 & 0.00 & 0.19 & 1.86 & 0.00 & 2.21 & 0.04 & 0.43 & 0.00 & 0.52 \\
\hline $\max$ & 7.00 & 6.00 & 16.70 & 18.20 & 60.69 & 6.06 & 78.89 & 3.29 & 9.99 & 1.72 & 13.19 \\
\hline mean & 1.81 & 1.95 & 11.31 & 2.32 & 12.42 & 1.60 & 16.34 & 0.49 & 2.61 & 0.44 & 3.54 \\
\hline med & 2.00 & 2.00 & 11.80 & 1.84 & 10.29 & 1.32 & 13.49 & 0.39 & 2.09 & 0.37 & 2.89 \\
\hline sd & 1.61 & 1.10 & 3.23 & 2.20 & 9.39 & 1.15 & 12.23 & 0.43 & 1.86 & 0.33 & 2.50 \\
\hline $\mathrm{se}$ & 0.16 & 0.11 & 0.32 & 0.22 & 0.94 & 0.12 & 1.22 & 0.04 & 0.19 & 0.03 & 0.25 \\
\hline CV (\%) & 88.74 & 56.16 & 28.58 & 95.10 & 75.61 & 72.01 & 74.83 & 88.20 & 71.08 & 74.70 & 70.59 \\
\hline $\mathrm{A}^{(1)}$ & $0.96^{*}$ & $0.71^{*}$ & $-1.73 *$ & $4.23 *$ & $2.29 *$ & $1.51 *$ & $2.25 *$ & $3.39 *$ & $1.76^{*}$ & $1.53^{*}$ & $1.74 *$ \\
\hline $\mathrm{K}^{(2)}$ & $0.98^{*}$ & $0.69^{\mathrm{ns}}$ & $4.22 *$ & $27.01 *$ & $7.65^{*}$ & $3.22 *$ & $7.34 *$ & $18.07 *$ & $3.71 *$ & $3.06^{*}$ & $3.51^{*}$ \\
\hline p-value & 0.01 & 0.00 & 0.01 & 0.00 & 0.04 & 0.18 & 0.01 & 0.01 & 0.03 & 0.15 & 0.04 \\
\hline \multicolumn{12}{|c|}{ Sowing time 2 (May 25, 2016) } \\
\hline $\min$ & 0.00 & 0.00 & 0.00 & 0.55 & 6.74 & 0.00 & 8.58 & 0.16 & 0.90 & 0.00 & 2.06 \\
\hline $\max$ & 13.00 & 13.00 & 22.40 & 27.93 & 132.56 & 17.37 & 175.22 & 4.28 & 19.95 & 4.60 & 28.83 \\
\hline mean & 4.08 & 4.35 & 14.13 & 9.28 & 41.14 & 4.74 & 55.17 & 1.61 & 7.65 & 1.32 & 10.58 \\
\hline med & 4.00 & 4.00 & 14.50 & 7.97 & 35.56 & 3.94 & 48.58 & 1.48 & 7.09 & 1.15 & 9.94 \\
\hline sd & 2.30 & 2.24 & 3.41 & 5.61 & 24.04 & 3.11 & 32.06 & 0.90 & 4.30 & 0.85 & 5.90 \\
\hline $\mathrm{se}$ & 0.23 & 0.22 & 0.34 & 0.56 & 2.40 & 0.31 & 3.21 & 0.09 & 0.43 & 0.09 & 0.59 \\
\hline CV (\%) & 56.46 & 51.49 & 24.14 & 60.44 & 58.42 & 65.67 & 58.11 & 56.34 & 56.26 & 64.50 & 55.75 \\
\hline $\mathrm{A}^{(1)}$ & $0.93^{*}$ & $0.68^{*}$ & $-1.96^{*}$ & $1.14^{*}$ & $1.20^{*}$ & $1.18^{*}$ & $1.22 *$ & $0.85^{*}$ & $0.78^{*}$ & $0.97^{*}$ & $0.81^{*}$ \\
\hline $\mathrm{K}^{(2)}$ & $1.48^{*}$ & $1.59^{*}$ & $7.25^{*}$ & $1.28 *$ & $1.87 *$ & $2.30^{*}$ & $1.89 *$ & $0.11^{\mathrm{ns}}$ & $0.23^{\mathrm{ns}}$ & $1.29 *$ & $0.33^{\mathrm{ns}}$ \\
\hline $\mathrm{p}$-value & 0.02 & 0.03 & 0.18 & 0.09 & 0.06 & 0.10 & 0.05 & 0.24 & 0.51 & 0.13 & 0.24 \\
\hline \multicolumn{12}{|c|}{ Sowing time 3 (June 7, 2016) } \\
\hline $\min$ & 0.00 & 1.00 & 7.00 & 0.39 & 4.69 & 0.17 & 5.61 & 0.10 & 0.95 & 0.03 & 1.34 \\
\hline $\max$ & 11.00 & 6.00 & 19.80 & 7.82 & 59.20 & 6.19 & 72.43 & 2.04 & 14.55 & 2.13 & 18.04 \\
\hline mean & 2.28 & 2.66 & 13.18 & 2.78 & 20.72 & 2.13 & 25.63 & 0.74 & 5.14 & 0.69 & 6.57 \\
\hline med & 2.00 & 3.00 & 13.20 & 2.53 & 19.61 & 2.00 & 24.46 & 0.67 & 4.77 & 0.62 & 6.12 \\
\hline $\mathrm{sd}$ & 1.54 & 1.03 & 2.38 & 1.58 & 10.05 & 1.18 & 12.42 & 0.41 & 2.78 & 0.43 & 3.50 \\
\hline $\mathrm{se}$ & 0.15 & 0.10 & 0.24 & 0.16 & 1.01 & 0.12 & 1.24 & 0.04 & 0.28 & 0.04 & 0.35 \\
\hline CV (\%) & 67.45 & 38.61 & 18.09 & 56.77 & 48.53 & 55.50 & 48.46 & 55.16 & 54.14 & 62.94 & 53.36 \\
\hline $\mathrm{A}^{(1)}$ & $2.47^{*}$ & $0.27^{\mathrm{ns}}$ & $0.04^{\mathrm{ns}}$ & $1.10^{*}$ & $1.00 *$ & $1.04^{*}$ & $1.00 *$ & $0.96^{*}$ & $1.17^{*}$ & $1.08^{*}$ & $1.11^{*}$ \\
\hline $\mathrm{K}^{(2)}$ & $11.17^{*}$ & $0.46^{\mathrm{ns}}$ & $0.14^{\mathrm{ns}}$ & $1.26^{*}$ & $1.61^{*}$ & $1.10^{*}$ & $1.52 *$ & $0.69^{\mathrm{ns}}$ & $1.53^{*}$ & $0.95^{*}$ & $1.34^{*}$ \\
\hline p-value & 0.00 & 0.00 & 0.98 & 0.05 & 0.46 & 0.26 & 0.39 & 0.07 & 0.18 & 0.22 & 0.16 \\
\hline \multicolumn{12}{|c|}{ Sowing time 4 (June 22, 2016) } \\
\hline $\min$ & 0.00 & 1.00 & 4.50 & 0.41 & 1.52 & 0.06 & 1.99 & 0.09 & 0.27 & 0.01 & 0.37 \\
\hline $\max$ & 6.00 & 6.00 & 17.40 & 7.43 & 40.05 & 5.74 & 50.12 & 2.21 & 11.10 & 1.58 & 14.30 \\
\hline mean & 1.83 & 2.53 & 12.76 & 2.63 & 17.24 & 1.83 & 21.70 & 0.76 & 4.58 & 0.59 & 5.92 \\
\hline med & 2.00 & 2.00 & 12.95 & 2.20 & 14.83 & 1.53 & 18.71 & 0.65 & 4.04 & 0.51 & 5.17 \\
\hline sd & 1.13 & 1.06 & 2.58 & 1.53 & 8.63 & 1.08 & 10.93 & 0.41 & 2.47 & 0.36 & 3.15 \\
\hline $\mathrm{se}$ & 0.11 & 0.11 & 0.26 & 0.15 & 0.86 & 0.11 & 1.09 & 0.04 & 0.25 & 0.04 & 0.32 \\
\hline CV (\%) & 61.67 & 41.84 & 20.23 & 57.98 & 50.02 & 59.28 & 50.36 & 54.30 & 54.02 & 61.46 & 53.28 \\
\hline $\mathrm{A}^{(1)}$ & $1.03^{*}$ & $0.94 *$ & $-0.76^{*}$ & $1.18^{*}$ & $0.92 *$ & $1.03^{*}$ & $0.95^{*}$ & $1.15^{*}$ & $0.90^{*}$ & $0.97^{*}$ & $0.93 *$ \\
\hline $\mathrm{K}^{(2)}$ & $2.07 *$ & $1.35^{*}$ & $0.83^{\mathrm{ns}}$ & $0.91^{\mathrm{ns}}$ & $0.17^{\mathrm{ns}}$ & $0.95^{*}$ & $0.27^{\mathrm{ns}}$ & $1.14 *$ & $0.23^{\mathrm{ns}}$ & $0.40^{\mathrm{ns}}$ & $0.32^{\mathrm{ns}}$ \\
\hline p-value & 0.00 & 0.00 & 0.48 & 0.01 & 0.01 & 0.01 & 0.01 & 0.01 & 0.07 & 0.01 & 0.07 \\
\hline
\end{tabular}




\begin{tabular}{lllllllllllll}
\hline \multicolumn{2}{l}{$\begin{array}{l}\text { Sowing time } \\
\text { min }\end{array}$} & (July 4, 2016) & & & & & & & & \\
max & 6.00 & 0.00 & 0.00 & 0.09 & 1.41 & 0.00 & 1.62 & 0.03 & 0.32 & 0.00 & 0.38 \\
mean & 1.08 & 4.00 & 15.90 & 5.39 & 29.04 & 4.09 & 36.88 & 1.49 & 7.29 & 1.26 & 9.53 \\
med & 1.00 & 1.00 & 10.50 & 1.18 & 8.33 & 0.83 & 11.01 & 0.40 & 2.01 & 0.30 & 2.77 \\
sd & 1.16 & 0.96 & 3.18 & 1.02 & 6.28 & 0.82 & 7.95 & 0.29 & 1.68 & 0.27 & 2.17 \\
se & 0.12 & 0.10 & 0.32 & 0.10 & 0.63 & 0.08 & 0.79 & 0.03 & 0.17 & 0.03 & 0.22 \\
$\mathrm{CV}(\%)$ & 107.47 & 55.94 & 30.61 & 70.75 & 64.60 & 73.96 & 64.69 & 63.27 & 65.01 & 71.47 & 63.65 \\
$\mathrm{~A}^{(1)}$ & $1.27^{*}$ & $0.62^{*}$ & $-1.29^{*}$ & $1.30^{*}$ & $1.04^{*}$ & $1.45^{*}$ & $1.09^{*}$ & $1.04^{*}$ & $0.90^{*}$ & $1.22^{*}$ & $0.90^{*}$ \\
$\mathrm{~K}^{(2)}$ & $2.27^{*}$ & $-0.41^{\mathrm{ns}}$ & $2.87^{*}$ & $1.81^{*}$ & $0.61^{\mathrm{ns}}$ & $2.14^{*}$ & $0.73^{\mathrm{ns}}$ & $1.08^{*}$ & $0.22^{\mathrm{ns}}$ & $1.34^{*}$ & $0.18^{\mathrm{ns}}$ \\
$\mathrm{p}$-value & 0.00 & 0.00 & 0.03 & 0.05 & 0.09 & 0.00 & 0.08 & 0.17 & 0.02 & 0.01 & 0.08 \\
\hline
\end{tabular}

Note. $^{(1)} *$ Asymmetry differs from zero, using the t-test, at a $5 \%$ probability level. ns Non-significant. ${ }^{(2)} *$ kurtosis differs from zero by means of the t-test, at a $5 \%$ probability level. ns Non-significant.

In the cultivar BRS Progresso, of 11 traits, ten, eight, eight, four and six traits, in times 1, 2, 3, 4 and 5, respectively, have leptokurtic behavior, i.e., there is a higher concentration of values around the central value. Whereas in the cultivar Temprano, eight, eleven, two, eleven and eight of the eleven traits in times 1, 2, 3, 4 and 5 , respectively, showed leptokurtic behavior.

Table 2. Minimum (min), maximum (max), mean, median (med), standard deviation (sd), standard error (se), coefficient of variation (CV, in \%), asymmetry (A), kurtosis (K) and p-value of the traits: number of tillers (NT); number of ears (NE); length of ear (LE, in $\mathrm{cm}$ ); fresh matter of leaf (FML, in g); fresh matter of stalk (FMS, in g); fresh matter of ear (FME, in g); fresh matter of plant (FMP = FML + FMS + FME, in g); dry matter of leaf (DML, in g); dry matter of stalk (DMS, in g); dry matter of ear (DME, in g); and dry matter of plant (DMP = DML + DMS + DME, in g) in the sowing times of rye Temprano

\begin{tabular}{|c|c|c|c|c|c|c|c|c|c|c|c|}
\hline Statistic & NT & $\mathrm{NE}$ & LE & FML & FMS & FME & FMP & DML & DMS & DME & DMP \\
\hline \multicolumn{12}{|c|}{ Sowing time 1 (May 3, 2016) } \\
\hline $\min$ & 0.00 & 0.00 & 0.00 & 0.02 & 2.67 & 0.00 & 2.98 & 0.01 & 0.79 & 0.00 & 0.91 \\
\hline $\max$ & 9.00 & 7.00 & 16.80 & 5.88 & 45.93 & 6.19 & 53.01 & 1.56 & 12.99 & 2.27 & 15.27 \\
\hline mean & 1.72 & 2.40 & 11.47 & 0.86 & 13.95 & 1.74 & 16.55 & 0.26 & 4.48 & 0.57 & 5.32 \\
\hline med & 2.00 & 2.00 & 11.40 & 0.64 & 12.33 & 1.53 & 14.64 & 0.20 & 3.77 & 0.49 & 4.43 \\
\hline sd & 1.58 & 1.38 & 2.48 & 0.88 & 8.18 & 1.08 & 9.65 & 0.25 & 2.64 & 0.38 & 3.13 \\
\hline se & 0.16 & 0.14 & 0.25 & 0.09 & 0.82 & 0.11 & 0.96 & 0.03 & 0.26 & 0.04 & 0.31 \\
\hline CV (\%) & 92.05 & 57.42 & 21.63 & 101.98 & 58.63 & 62.10 & 58.28 & 94.64 & 58.84 & 66.55 & 58.79 \\
\hline $\mathrm{A}^{(1)}$ & $1.36^{*}$ & $0.97^{*}$ & $-0.93 *$ & $2.64 *$ & $1.03 *$ & $1.25^{*}$ & $0.96^{*}$ & $2.12^{*}$ & $0.96^{*}$ & $1.57^{*}$ & $0.95^{*}$ \\
\hline $\mathrm{K}^{(2)}$ & $3.58^{*}$ & $0.83^{\mathrm{ns}}$ & $3.61^{*}$ & $10.69^{*}$ & $1.32 *$ & $2.19^{*}$ & $0.99 *$ & $6.78^{*}$ & $0.83^{\mathrm{ns}}$ & $3.75^{*}$ & $0.71^{\mathrm{ns}}$ \\
\hline p-value & 0.01 & 0.00 & 0.96 & 0.00 & 0.19 & 0.21 & 0.23 & 0.02 & 0.04 & 0.10 & 0.10 \\
\hline \multicolumn{12}{|c|}{ Sowing time 2 (May 25, 2016) } \\
\hline $\min$ & 0.00 & 0.00 & 0.00 & 0.45 & 4.23 & 0.00 & 5.26 & 0.15 & 0.89 & 0.00 & 1.22 \\
\hline $\max$ & 33.00 & 23.00 & 20.00 & 51.70 & 147.58 & 17.01 & 185.35 & 12.60 & 39.64 & 5.64 & 51.73 \\
\hline mean & 5.33 & 4.03 & 13.45 & 6.25 & 39.07 & 4.45 & 49.77 & 1.78 & 10.50 & 1.45 & 13.72 \\
\hline med & 4.00 & 3.00 & 14.50 & 4.97 & 34.41 & 3.76 & 43.79 & 1.48 & 9.07 & 1.20 & 11.34 \\
\hline sd & 4.84 & 3.44 & 4.55 & 6.22 & 26.32 & 3.55 & 33.47 & 1.59 & 7.71 & 1.24 & 9.85 \\
\hline se & 0.48 & 0.34 & 0.45 & 0.62 & 2.63 & 0.36 & 3.35 & 0.16 & 0.77 & 0.12 & 0.98 \\
\hline CV (\%) & 90.75 & 85.34 & 33.82 & 99.56 & 67.38 & 79.86 & 67.25 & 89.53 & 73.46 & 85.58 & 71.77 \\
\hline $\mathrm{A}^{(1)}$ & $3.04 *$ & $2.24 *$ & $-1.95^{*}$ & $4.49 *$ & $1.30^{*}$ & $1.16^{*}$ & $1.32 *$ & $3.79 *$ & $1.45^{*}$ & $1.23 *$ & $1.39 *$ \\
\hline $\mathrm{K}^{(2)}$ & $12.89^{*}$ & $8.84^{*}$ & $3.75^{*}$ & $29.02 *$ & $2.36^{*}$ & $1.42 *$ & $2.36^{*}$ & $21.65^{*}$ & $2.35^{*}$ & $1.49 *$ & $2.20 *$ \\
\hline p-value & 0.00 & 0.01 & 0.00 & 0.00 & 0.15 & 0.22 & 0.18 & 0.00 & 0.15 & 0.11 & 0.15 \\
\hline \multicolumn{12}{|c|}{ Sowing time 3 (June 7, 2016) } \\
\hline $\min$ & 0.00 & 0.00 & 0.00 & 0.20 & 2.91 & 0.00 & 3.40 & 0.03 & 0.83 & 0.00 & 0.96 \\
\hline $\max$ & 8.00 & 6.00 & 19.90 & 9.02 & 51.29 & 7.72 & 63.83 & 2.20 & 17.40 & 2.51 & 21.56 \\
\hline mean & 2.67 & 2.51 & 11.75 & 2.28 & 18.59 & 2.40 & 23.27 & 0.66 & 5.43 & 0.75 & 6.84 \\
\hline med & 2.50 & 2.00 & 12.10 & 1.92 & 15.92 & 1.70 & 20.28 & 0.60 & 4.44 & 0.58 & 5.55 \\
\hline
\end{tabular}




\begin{tabular}{|c|c|c|c|c|c|c|c|c|c|c|c|}
\hline sd & 1.91 & 1.52 & 3.32 & 1.77 & 11.23 & 1.81 & 13.91 & 0.46 & 3.53 & 0.61 & 4.35 \\
\hline $\mathrm{se}$ & 0.19 & 0.15 & 0.33 & 0.18 & 1.12 & 0.18 & 1.39 & 0.05 & 0.35 & 0.06 & 0.44 \\
\hline CV (\%) & 71.43 & 60.59 & 28.30 & 77.67 & 60.40 & 75.58 & 59.78 & 69.96 & 65.04 & 81.50 & 63.65 \\
\hline $\mathrm{A}^{(1)}$ & $0.71 *$ & $0.63 *$ & $-1.17^{*}$ & $1.28 *$ & $0.95^{*}$ & $1.05^{*}$ & $0.94 *$ & $1.03 *$ & $1.11 *$ & $1.06^{*}$ & $1.08^{*}$ \\
\hline $\mathrm{K}^{(2)}$ & $0.03^{\mathrm{ns}}$ & $-0.44^{\mathrm{ns}}$ & $3.20 *$ & $1.54 *$ & $0.34^{\mathrm{ns}}$ & $0.46^{\mathrm{ns}}$ & $0.40^{\mathrm{ns}}$ & $0.85^{\mathrm{ns}}$ & $0.79^{\mathrm{ns}}$ & $0.45^{\mathrm{ns}}$ & $0.73^{\mathrm{ns}}$ \\
\hline p-value & 0.01 & 0.00 & 0.53 & 0.04 & 0.07 & 0.01 & 0.13 & 0.20 & 0.02 & 0.03 & 0.01 \\
\hline \multicolumn{12}{|c|}{ Sowing time 4 (June 22, 2016) } \\
\hline $\min$ & 0.00 & 0.00 & 0.00 & 0.05 & 4.26 & 0.00 & 5.21 & 0.01 & 0.97 & 0.00 & 1.18 \\
\hline $\max$ & 16.00 & 8.00 & 17.80 & 8.83 & 86.57 & 8.34 & 96.84 & 1.90 & 17.12 & 2.68 & 20.90 \\
\hline mean & 2.71 & 2.71 & 12.02 & 0.90 & 20.82 & 2.27 & 23.98 & 0.38 & 5.25 & 0.70 & 6.33 \\
\hline med & 2.00 & 3.00 & 12.30 & 0.59 & 17.40 & 2.04 & 19.92 & 0.26 & 4.15 & 0.57 & 5.07 \\
\hline sd & 2.28 & 1.50 & 3.30 & 1.18 & 13.66 & 1.55 & 15.57 & 0.30 & 3.34 & 0.51 & 3.98 \\
\hline se & 0.23 & 0.15 & 0.33 & 0.12 & 1.37 & 0.16 & 1.56 & 0.03 & 0.33 & 0.05 & 0.40 \\
\hline $\mathrm{CV}(\%)$ & 84.29 & 55.33 & 27.48 & 131.72 & 65.62 & 68.56 & 64.91 & 79.14 & 63.59 & 72.52 & 62.85 \\
\hline $\mathrm{A}^{(1)}$ & $2.95 *$ & $0.84 *$ & $-1.87 *$ & $4.18^{*}$ & $2.15^{*}$ & $1.43^{*}$ & $2.08 *$ & $2.12 *$ & $1.53 *$ & $1.55^{*}$ & $1.52 *$ \\
\hline $\mathrm{K}^{(2)}$ & $12.52 *$ & $1.45^{*}$ & $5.09 *$ & $22.88^{*}$ & $6.20 *$ & $3.04 *$ & $5.68 *$ & $6.84^{*}$ & $2.33 *$ & $3.24 *$ & $2.28 *$ \\
\hline p-value & 0.00 & 0.00 & 0.07 & 0.00 & 0.03 & 0.24 & 0.02 & 0.01 & 0.01 & 0.04 & 0.02 \\
\hline \multicolumn{12}{|c|}{ Sowing time 5 (July 4, 2016) } \\
\hline $\min$ & 0.00 & 0.00 & 0.00 & 0.09 & 2.09 & 0.00 & 2.44 & 0.03 & 0.71 & 0.00 & 0.84 \\
\hline $\max$ & 9.00 & 5.00 & 15.60 & 6.08 & 30.91 & 4.66 & 38.12 & 1.61 & 8.18 & 1.75 & 10.33 \\
\hline mean & 2.32 & 1.90 & 10.61 & 0.92 & 10.45 & 1.34 & 12.71 & 0.36 & 3.30 & 0.50 & 4.16 \\
\hline med & 2.00 & 2.00 & 10.60 & 0.58 & 9.61 & 1.17 & 11.73 & 0.31 & 2.97 & 0.41 & 3.90 \\
\hline $\mathrm{sd}$ & 2.01 & 1.03 & 3.01 & 1.09 & 5.19 & 0.89 & 6.24 & 0.27 & 1.65 & 0.39 & 2.07 \\
\hline $\mathrm{se}$ & 0.20 & 0.10 & 0.30 & 0.11 & 0.52 & 0.09 & 0.62 & 0.03 & 0.17 & 0.04 & 0.21 \\
\hline CV (\%) & 86.83 & 54.20 & 28.38 & 117.73 & 49.65 & 66.11 & 49.09 & 76.57 & 50.06 & 76.97 & 49.72 \\
\hline $\mathrm{A}^{(1)}$ & $1.45^{*}$ & $0.66^{*}$ & $-1.61^{*}$ & $2.81 *$ & $1.42 *$ & $1.22 *$ & $1.40 *$ & $2.23 *$ & $0.88 *$ & $1.32 *$ & $0.87 *$ \\
\hline $\mathrm{K}^{(2)}$ & $2.04 *$ & $0.41^{\mathrm{ns}}$ & $4.29 *$ & $8.96^{*}$ & $3.36^{*}$ & $2.04 *$ & $3.47 *$ & $6.76^{*}$ & $0.42^{\mathrm{ns}}$ & $1.84^{*}$ & $0.48^{\mathrm{ns}}$ \\
\hline $\mathrm{p}$-value & 0.00 & 0.00 & 0.10 & 0.00 & 0.14 & 0.21 & 0.34 & 0.01 & 0.12 & 0.15 & 0.08 \\
\hline
\end{tabular}

Note. $^{\left({ }^{(1)}\right.} *$ Asymmetry differs from zero, using the t-test, at a $5 \%$ probability level. ns Non-significant. ${ }^{(2)} *$ Kurtosis differs from zero by means of the t-test, at a 5\% probability level. ns Non-significant.

For the cultivar BRS Progresso, the CV (\%) ranged between 18.09 and $107.47 \%$ and to Temprano cultivar, between 21.63 and $131.72 \%$. The LE exhibited a lower variability among the traits for both cultivars, which suggests a smaller sample size. In the cultivar BRS Progresso the feature that showed greater variability was the NT and to Temprano cultivar, the trait FML.

In the cultivar BRS Progresso, it was found that in the first and last sowing times, the variability of the data was greater than in intermediate sowing times. However, the cultivar Temprano did not exhibit this behavior pattern. Therefore, there were differences among the traits, cultivars and sowing times, suggesting that this variation is also seen in the sample size. The scenario of wide variability in the database is important, giving credibility to the study (Cargnelutti Filho et al., 2015a).

The deviations of the asymmetry and kurtosis contributed to the removal of the data in relation to the curve of normal distribution in nine, two, two, eight and six traits, in the cultivar BRS Progresso and five, five, seven, nine and four in the cultivar Temprano, in times one, two, three, four and five, respectively. In these cases, with a probability distribution is unknown, the bootstrap resampling technique with replacement is recommended (Ferreira, 2009).

The sample size to estimate the mean of each trait, with amplitude of the confidence interval of $95 \%\left(\mathrm{ACI}_{95 \%}\right)$ of $5 \%$ of the mean, ranged from 194 to more than 1,000 plants in cultivar BRS Progresso (Table 3) and 282 of the more than 1,000 plants in the cultivar Temprano (Table 4). In both cultivars and sowing times, for the trait LE, smaller sample sizes were found than the other traits (Figures 1A and 1C). Larger sample size is necessary for the trait NT in the cultivar BRS Progresso (Figure 1B) and to FML in cultivar Temprano (Figure 1D). These results are in accordance with the scenario of increasing variability observed for the traits NE, FMP, FMS, DMP, DMS, FME, DME, DML, in the FML, in this order (Tables 1 and 2). 

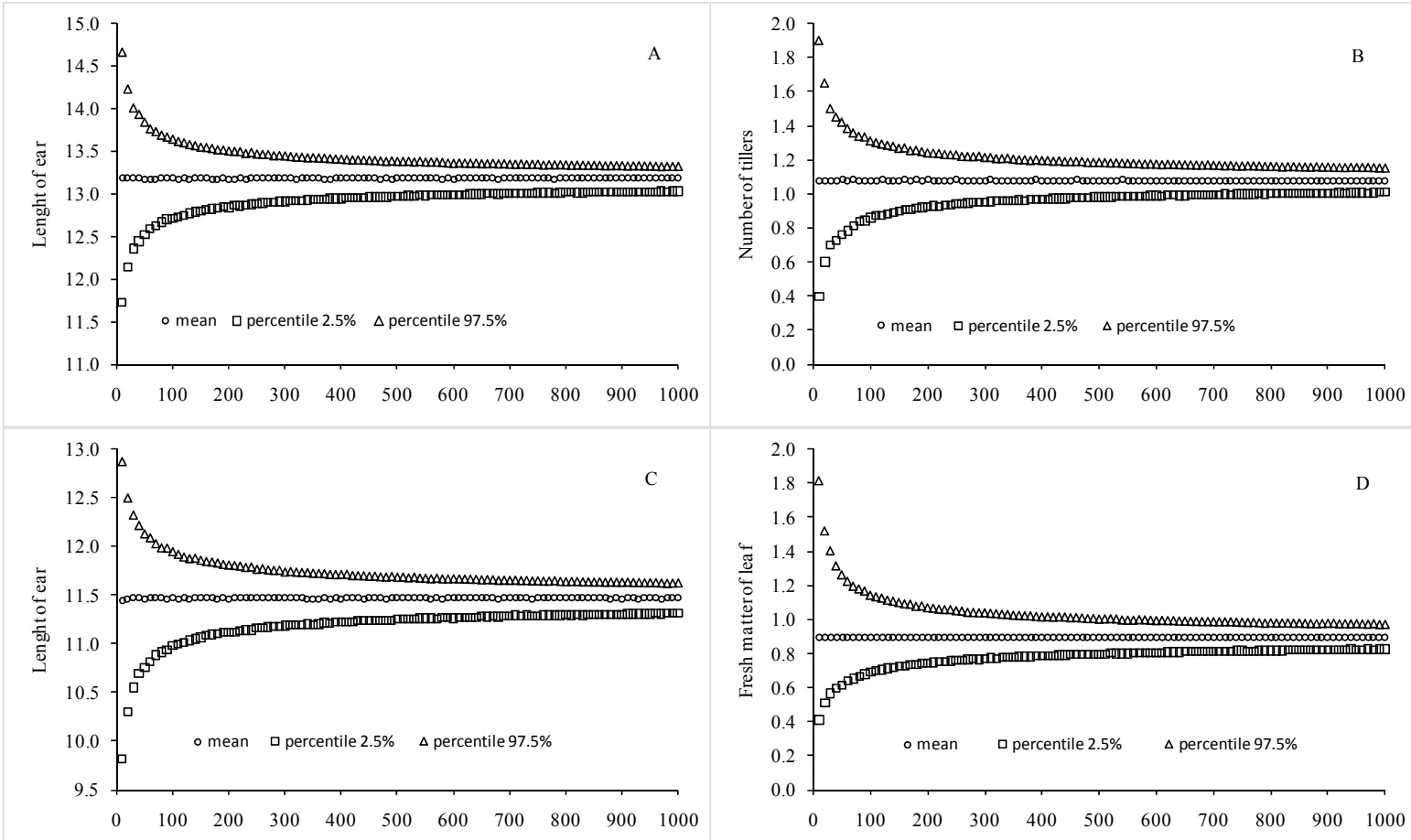

Figure 1. Percentile $2.5 \%$, mean and percentile $97.5 \%$ of the 1,000 estimates of the mean of traits lenght of ear (LE, in cm) (A) and the number of tillers (NT) (B) in sowing times three and five, respectively, of cultivar BRS Progresso, lenght of ear (LE, in $\mathrm{cm}$ ) (C) and fresh matter of leaf (FML, in g) (D) in sowing times five and four, respectively, of the cultivar Temprano, for sample sizes of $10,20,30, \ldots, 1,000$ plants of rye

Table 3. Sample size (number of plants) for the mean trait estimation, number of tillers (NT); number of ears (NE); length of ear (LE, in cm); fresh matter of leaf (FML, in g); fresh matter of stalk (FMS, in g); fresh matter of ear (FME, in g); fresh matter of plant (FMP = FML + FMS + FME, in g); dry matter of leaf (DML, in g); dry matter of stalk (DMS, in g); dry matter of ear (DME, in g); and dry matter of plant (DMP = DML + DMS + DME, in $\mathrm{g}$ ) of the cultivar BRS Progresso, for the amplitude of the confidence interval $\left(\mathrm{ACI}_{95 \%}\right)$ of $5,10,15,20$, 25,30 and $35 \%$ of the mean, at sowing times

\begin{tabular}{|c|c|c|c|c|c|c|c|c|c|c|c|}
\hline ACI95\% & NT & $\mathrm{NE}$ & LE & FML & FMS & FME & FMP & DML & DMS & DME & DMP \\
\hline \multicolumn{12}{|c|}{ Sowing time 1 (May 3, 2016) } \\
\hline $5 \%$ & $>1000$ & $>1000$ & 492 & $>1000$ & $>1000$ & $>1000$ & $>1000$ & $>1000$ & $>1000$ & $>1000$ & $>1000$ \\
\hline $10 \%$ & $>1000$ & 467 & 121 & $>1000$ & 837 & 768 & 836 & $>1000$ & 731 & 827 & 733 \\
\hline $15 \%$ & 523 & 210 & 55 & 595 & 368 & 339 & 368 & 520 & 339 & 371 & 324 \\
\hline $20 \%$ & 292 & 116 & 31 & 333 & 216 & 191 & 210 & 290 & 194 & 215 & 188 \\
\hline $25 \%$ & 188 & 77 & 20 & 220 & 137 & 126 & 131 & 187 & 121 & 136 & 119 \\
\hline $30 \%$ & 131 & 53 & 15 & 151 & 93 & 87 & 96 & 132 & 86 & 95 & 84 \\
\hline $35 \%$ & 97 & 37 & 10 & 107 & 70 & 63 & 70 & 98 & 64 & 70 & 61 \\
\hline \multicolumn{12}{|c|}{ Sowing time 2 (May 25, 2016) } \\
\hline $5 \%$ & $>1000$ & $>1000$ & 340 & $>1000$ & $>1000$ & $>1000$ & $>1000$ & $>1000$ & $>1000$ & $>1000$ & $>1000$ \\
\hline $10 \%$ & 476 & 389 & 89 & 534 & 506 & 630 & 492 & 474 & 468 & 617 & 468 \\
\hline $15 \%$ & 210 & 177 & 39 & 246 & 227 & 284 & 229 & 215 & 215 & 271 & 207 \\
\hline $20 \%$ & 121 & 102 & 22 & 137 & 128 & 162 & 129 & 120 & 122 & 155 & 115 \\
\hline $25 \%$ & 75 & 63 & 15 & 86 & 82 & 105 & 82 & 76 & 77 & 101 & 74 \\
\hline $30 \%$ & 54 & 44 & 10 & 62 & 58 & 74 & 58 & 54 & 53 & 71 & 50 \\
\hline $35 \%$ & 40 & 33 & 8 & 45 & 42 & 55 & 43 & 39 & 39 & 51 & 39 \\
\hline \multicolumn{12}{|c|}{ Sowing time 3 (June 7,2016$)$} \\
\hline $5 \%$ & $>1000$ & 884 & 194 & $>1000$ & $>1000$ & $>1000$ & $>1000$ & $>1000$ & $>1000$ & $>1000$ & $>1000$ \\
\hline
\end{tabular}




\begin{tabular}{llllllllllll}
\hline $10 \%$ & 681 & 222 & 51 & 484 & 355 & 461 & 345 & 463 & 433 & 602 & 423 \\
$15 \%$ & 306 & 98 & 23 & 209 & 155 & 203 & 159 & 207 & 197 & 265 & 191 \\
$20 \%$ & 171 & 57 & 13 & 120 & 91 & 117 & 87 & 116 & 110 & 151 & 108 \\
$25 \%$ & 111 & 38 & 9 & 78 & 56 & 74 & 57 & 74 & 70 & 96 & 70 \\
$30 \%$ & 76 & 27 & 6 & 53 & 41 & 52 & 40 & 51 & 51 & 68 & 48 \\
$35 \%$ & 58 & 18 & 5 & 40 & 30 & 40 & 29 & 38 & 36 & 50 & 36 \\
\hline Sowing time & $4(J u n e$ & 22, & $2016)$ & & & & & & & & \\
$5 \%$ & $>1000$ & $>1000$ & 248 & $>1000$ & $>1000$ & $>1000$ & $>1000$ & $>1000$ & $>1000$ & $>1000$ & $>1000$ \\
$10 \%$ & 565 & 261 & 62 & 504 & 375 & 512 & 384 & 446 & 436 & 567 & 425 \\
$15 \%$ & 255 & 116 & 28 & 224 & 172 & 234 & 170 & 201 & 197 & 250 & 185 \\
$20 \%$ & 145 & 65 & 16 & 129 & 94 & 133 & 94 & 112 & 110 & 143 & 109 \\
$25 \%$ & 92 & 40 & 10 & 81 & 61 & 88 & 62 & 72 & 70 & 92 & 68 \\
$30 \%$ & 64 & 29 & 8 & 58 & 43 & 61 & 44 & 48 & 49 & 64 & 49 \\
$35 \%$ & 48 & 23 & 6 & 43 & 32 & 44 & 32 & 37 & 36 & 47 \\
- Sowing time & 5 (July 4,2016$)$ & & & & & & & 36 \\
$5 \%$ & $>1000$ & $>1000$ & 550 & $>1000$ & $>1000$ & $>1000$ & $>1000$ & $>1000$ & $>1000$ & $>1000$ & $>1000$ \\
$10 \%$ & $>1000$ & 466 & 140 & 751 & 629 & 809 & 622 & 598 & 638 & 747 & 607 \\
$15 \%$ & 761 & 207 & 63 & 329 & 278 & 366 & 277 & 267 & 285 & 341 & 265 \\
$20 \%$ & 425 & 117 & 36 & 187 & 159 & 209 & 157 & 149 & 161 & 190 & 152 \\
$25 \%$ & 276 & 75 & 23 & 121 & 101 & 133 & 99 & 95 & 103 & 123 & 98 \\
$30 \%$ & 189 & 53 & 16 & 83 & 70 & 96 & 72 & 67 & 71 & 86 & 67 \\
$35 \%$ & 138 & 42 & 12 & 61 & 52 & 67 & 53 & 50 & 52 & 61 & 49 \\
\hline
\end{tabular}

Variability among traits, as to the size of the sample, was observed by Cargnelutti Filho et al. (2014) in the culture of forage turnip, Toebe et al. (2014) in the culture of maize and Silva et al. (2015) in the sunflower culture. Cargnelutti Filho et al. (2009), besides verifying variability in sample size among the traits, found differences regarding the sample size among soybean genotypes. This can also be described in the present study, once that for the cultivar BRS Progresso, in general, the sample sizes were smaller than for cultivar Temprano.

Table 4. Sample size (number of plants) for the mean trait estimation, number of tillers (NT); number of ears (NE); length of ear (LE, in cm); fresh matter of leaf (FML, in g); fresh matter of stalk (FMS, in g); fresh matter of ear (FME, in g); fresh matter of plant (FMP = FML + FMS + FME, in g); dry matter of leaf (DML, in g); dry matter of stalk (DMS, in g); dry matter of ear (DME, in g); and dry matter of plant (DMP = DML + DMS + DME, in $\mathrm{g}$ ) of the cultivar Temprano, for the amplitude of the confidence interval ( $\left.\mathrm{ACI}_{95 \%}\right)$ of 5, 10, 15, 20, 25, 30 and $35 \%$ of the mean, at sowing times

\begin{tabular}{llllllllllll}
\hline ACI95\% & NT & NE & LE & FML & FMS & FME & FMP & DML & DMS & DME & DMP \\
\hline $\begin{array}{l}\text { Sowing time } \\
\text { 1 (May 3, }\end{array}$ 2016) & & & & & & & & \\
$10 \%$ & $>1000$ & $>1000$ & 282 & $>1000$ & $>1000$ & $>1000$ & $>1000$ & $>1000$ & $>1000$ & $>1000$ & $>1000$ \\
$15 \%$ & $>1000$ & 489 & 70 & $>1000$ & 510 & 564 & 510 & $>1000$ & 512 & 667 & 510 \\
$20 \%$ & 562 & 220 & 32 & 683 & 226 & 258 & 228 & 588 & 233 & 296 & 232 \\
$25 \%$ & 320 & 120 & 18 & 385 & 130 & 148 & 127 & 337 & 129 & 168 & 133 \\
$30 \%$ & 202 & 81 & 12 & 246 & 85 & 93 & 84 & 217 & 83 & 107 & 82 \\
$35 \%$ & 138 & 56 & 9 & 171 & 60 & 65 & 59 & 150 & 60 & 75 & 60 \\
- Sowing time & 104 & 42 & 7 & 129 & 44 & 48 & 43 & 109 & 43 & 55 & 43 \\
$5 \%$ & $>1000$ & $>1000$ & 689 & $>1000$ & $>1000$ & $>1000$ & $>1000$ & $>1000$ & $>1000$ & $>1000$ & $>1000$ \\
$10 \%$ & $>1000$ & $>1000$ & 172 & $>1000$ & 669 & 948 & 683 & $>1000$ & 801 & $>1000$ & 762 \\
$15 \%$ & 543 & 480 & 75 & 648 & 301 & 421 & 296 & 529 & 360 & 482 & 344 \\
$20 \%$ & 307 & 275 & 44 & 371 & 170 & 240 & 170 & 299 & 201 & 275 & 193 \\
$25 \%$ & 196 & 177 & 28 & 237 & 110 & 152 & 110 & 194 & 129 & 177 & 123 \\
$30 \%$ & 134 & 123 & 20 & 160 & 78 & 107 & 74 & 130 & 89 & 123 & 86 \\
$35 \%$ & 102 & 90 & 14 & 122 & 55 & 77 & 58 & 100 & 68 & 87 & 65 \\
\hline
\end{tabular}




\begin{tabular}{|c|c|c|c|c|c|c|c|c|c|c|c|}
\hline \multicolumn{12}{|c|}{ Sowing time 3 (June 7, 2016) } \\
\hline $5 \%$ & $>1000$ & $>1000$ & 477 & $>1000$ & $>1000$ & $>1000$ & $>1000$ & $>1000$ & $>1000$ & $>1000$ & $>1000$ \\
\hline $10 \%$ & 756 & 548 & 119 & 895 & 543 & 857 & 538 & 732 & 632 & 989 & 582 \\
\hline $15 \%$ & 331 & 246 & 54 & 396 & 247 & 372 & 236 & 328 & 278 & 447 & 271 \\
\hline $20 \%$ & 190 & 143 & 31 & 228 & 139 & 212 & 132 & 185 & 158 & 250 & 151 \\
\hline $25 \%$ & 123 & 87 & 19 & 145 & 87 & 137 & 87 & 120 & 102 & 162 & 96 \\
\hline $30 \%$ & 87 & 63 & 14 & 100 & 63 & 96 & 62 & 83 & 72 & 113 & 67 \\
\hline $35 \%$ & 63 & 45 & 11 & 75 & 44 & 71 & 46 & 60 & 52 & 81 & 51 \\
\hline \multicolumn{12}{|c|}{ Sowing time 4 (June 22, 2016) } \\
\hline $5 \%$ & $>1000$ & $>1000$ & 451 & $>1000$ & $>1000$ & $>1000$ & $>1000$ & $>1000$ & $>1000$ & $>1000$ & $>1000$ \\
\hline $10 \%$ & $>1000$ & 443 & 116 & $>1000$ & 642 & 688 & 619 & 935 & 606 & 777 & 590 \\
\hline $15 \%$ & 479 & 205 & 51 & $>1000$ & 280 & 312 & 278 & 409 & 269 & 354 & 263 \\
\hline $20 \%$ & 265 & 115 & 28 & 642 & 164 & 173 & 159 & 232 & 152 & 198 & 147 \\
\hline $25 \%$ & 170 & 71 & 18 & 413 & 104 & 112 & 104 & 148 & 96 & 127 & 97 \\
\hline $30 \%$ & 114 & 53 & 13 & 285 & 73 & 80 & 71 & 107 & 69 & 91 & 66 \\
\hline $35 \%$ & 88 & 39 & 10 & 211 & 54 & 57 & 53 & 79 & 51 & 67 & 49 \\
\hline \multicolumn{12}{|c|}{ Sowing time 5 (July 4, 2016) } \\
\hline $5 \%$ & $>1000$ & $>1000$ & 474 & $>1000$ & $>1000$ & $>1000$ & $>1000$ & $>1000$ & $>1000$ & $>1000$ & $>1000$ \\
\hline $10 \%$ & $>1000$ & 436 & 122 & $>1000$ & 371 & 656 & 364 & 865 & 380 & 877 & 368 \\
\hline $15 \%$ & 506 & 193 & 55 & 899 & 165 & 291 & 164 & 384 & 164 & 399 & 162 \\
\hline $20 \%$ & 278 & 111 & 32 & 519 & 92 & 165 & 93 & 220 & 96 & 218 & 95 \\
\hline $25 \%$ & 178 & 74 & 20 & 325 & 60 & 105 & 58 & 144 & 60 & 144 & 60 \\
\hline $30 \%$ & 128 & 48 & 14 & 226 & 42 & 75 & 41 & 98 & 42 & 99 & 42 \\
\hline $35 \%$ & 92 & 35 & 10 & 171 & 31 & 54 & 31 & 72 & 32 & 74 & 31 \\
\hline
\end{tabular}

The sowing times influenced the sample size in both cultivars. In the BRS Progresso, the first and the last time of sowing had the largest sample size, which was not observed in the cultivar Temprano. In the culture of pigeonpea, Facco et al. (2015) found variability for the sample size among traits, between the period of evaluation of culture during its development and among crop years. This shows that before different environmental conditions each culture and cultivate respond in a way, that is why it is fundamental the presence of different scenarios to estimate the sample size.

The individual assessment of more than 1,000 plants in both cultivars in field experiments would be difficult to measure, because there are a large number of plants, requiring much labor and time of the researcher. To solve this problem, it is estimated sample sizes for $\mathrm{ACI}_{95 \%}$ of $10,15,20,25,30$ and $35 \%$ of the mean (Tables 3 and 4 ). Upon defining the size of the sample, the trait and the level of precision required should be taken into account. In case the researcher opts for a sample size with $\mathrm{ACI}_{95 \%}$ of $20 \%$ of the mean for all traits and the sowing times, for the cultivar BRS Progresso, 189 plants would need to be assessed, and to cultivar Temprano, 285 plants. Thus, following indications of Cargnelutti Filho et al. (2015b), for an experiment carried out in a randomized block design with four replications, 48 and 72 plants of cultivars BRS Progresso and Temprano, respectively would have to be assessed, in each repetition.

\section{Conclusions}

There is variability in the sample size to estimate the mean among the traits, cultivars and sowing times in the rye crop.

The measurement of 425, 276, 189 and 138 plants in cultivar BRS Progresso and 642, 413, 285 and 211 plants in cultivar Temprano, are enough to estimate the mean amplitude of the confidence interval of $95 \%$ maximum of $20,25,30$ and $35 \%$, respectively, for all the traits and sowing times.

\section{References}

Basche, A. D., Kaspar, T. C., Archontoulis, S. V., Jaymes, D. B., Sauer, T. J., Parkin, T. B., \& Miguez, F. E. (2016). Soil water improvements with the long-term use of a winter rye cover crop. Agricultural Water Management, 172, 40-50. https://doi.org/10.1016/j.agwat.2016.04.006

Banzatto, D. A., \& Kronka, S. N. (2013). Experimentação agrícola (4th ed.). Jaboticabal: FUNEP. 
Brito, C. J., Grigoletto, M. E. da S., Nóbrega, O. de T., \& Córdova, C. (2016). Dimensionamento de amostras e o mito dos números mágicos: Ponto de vista. Revista Andaluza de Medicina del Deporte, 9, 29-31. https://doi.org/10.1016/j.ramd.2015.02.007

Bussab, W. de O., \& Morettin, P. A. (2013). Estatística Básica (8th ed.). São Paulo: Saraiva.

Campos, H. D. E. (1983). Estatística experimental não-paramétrica (4th ed.). Piracibaba: ESALQ.

Cargnelutti Filho, A., Alves, B. M., Burin, C., Kleinpaul, J. A., Neu, I. M. M., Silveira, D. L., ... Medeiros, L. B. (2015a). Tamanho de parcela e número de repetições em ervilhaca forrageira. Ciência Rural, 45, 1174-1182. https://doi.org/10.1590/0103-8478cr20141043

Cargnelutti Filho, A., Evangelista, D. H. R., Gonçalves, E. C. P., \& Storck, L. (2009). Tamanho de amostra de caracteres de genótipos de soja. Ciência Rural, 39, 983-991. https://doi.org/10.1590/S0103-8478200900 5000016

Cargnelutti Filho, A., Facco, G., Lúcio, A. D., Toebe, M., Burin, C., Fick, A. L., \& Neu, I. M. M. (2014). Tamanho de amostra para a estimação da média de caracteres morfológicos e produtivos de nabo forrageiro. Ciência Rural, 44, 223-227. https://doi.org/10.1590/S0103-84782014000200005

Cargnelutti Filho, A., Toebe, M., Alves, B. M., Burin, C., Santos, G. O., Facco, G., \& Neu, I. M. M. (2015b). Dimensionamento amostral para avaliar caracteres morfológicos e produtivos de aveia preta em épocas de avaliação. Ciência Rural, 45, 9-13. https://doi.org/10.1590/0103-8478cr20140504

Facco, G., Cargnelutti Filho, A., Lúcio, A. D., Santos,G. O. dos, Stefanello, R. B., Alves, B. M., ... Kleinpaul, J. A. (2015). Sample size for morphological traits of pigeonpea. Semina: Ciências Agrárias, 36, 4151-4164. https://doi.org/10.5433/1679-0359.2015v36n6Supl2p4151

Ferreira, D. F. (2009). Estatística básica (2nd ed.). Lavras: UFLA.

Heldwein, A. B., Buriol, G. A., \& Streck, N. A. (2009). O clima de Santa Maria. Ciência \& Ambiente, 38, 43-58.

Martinez-Feria, R. A., Dietzel, R., Liebman, M., Helmers, M. J., \& Archontoulis, S. V. (2016). Rye cover crop effects on maize: A system-level analysis. Field Crops Research, 196, 145-159. http://doi.org/10.1016/ j.fcr.2016.06.016

Pantoja, J. L., Woli, K. P., Sawyer, J. E., \& Barker, D. W. (2016). Winter rye cover crop biomass production, degradation, and nitrogen recycling. Agronomy Journal, 108, 841-853. https://doi.org/10.2134/agronj 2015.0336

Ramírez, I. C., Barrera, C. J., \& Correa, J. C. (2013). Efecto del tamaño de muestra y el número de réplicas bootstrap. Ingeniería y Competitividad, 15, 93-101.

R Core Team. (2017). R: A language and environment for statistical computing. R Foundation for Statistical Computing, Vienna, Austria. Retrieved from https://www.R-project.org

Santos, H. G. dos, Almeida, J. A., \& Oliveira, J. B. (2013). Sistema Brasileiro de Classificação de Solos (3rd ed.). Brasília: Embrapa.

Silva, P. S. L., Santos, L. E. B., Oliveira, V. R., Sousa, R. P., \& Fernandes, P. L. O. (2015). Sample size and sampling method for evaluation of characteristics of the sunflower. Revista Ciência Agronômica, 46, 144-154. https://doi.org/10.1590/S1806-66902015000100017

Storck, L., Garcia, D. C., Lopes, S. J., \& Estefanel, V. (2016). Experimentação vegetal (3rd ed.). Santa Maria: UFSM.

Toebe, M., Cargnelutti Filho, A., Burin, C., Casarotto, G., \& Haesbaert, F. M. (2014). Tamanho de amostra para a estimação da média e do coeficiente de variação em milho. Pesquisa Agropecuária Brasileira, 49, 860-871. https://doi.org/10.1590/S0100-204X2014001100005

\section{Copyrights}

Copyright for this article is retained by the author(s), with first publication rights granted to the journal.

This is an open-access article distributed under the terms and conditions of the Creative Commons Attribution license (http://creativecommons.org/licenses/by/4.0/). 